\title{
Cancer Survivorship Care: A perspective from an Occupational- Participation Approach
}

\section{Siew Yim Loh ${ }^{1 *}$ and Hans Jonsson ${ }^{2}$}

${ }^{1}$ Department of Rehabilitation, Faculty of Medicine, University of Malaya, 50603 Kuala Lumpur, Malaysia

${ }^{2}$ Department of Occupational Therapy, Karolinska Institute, Sweden

\begin{abstract}
The burden of cancer taking a form of a chronic illness warrants a shift in the health care delivery to address the many neglected functional impacts (physical-psychosocial and occupational dysfunctions) from cancer. Balancing the current traditional medical care (that focuses on the tumour/illness), with the essential non-medical tasks of dayto-day participation and self-care tasks are both needed for cancer conditions that now requires care over indefinite period. This paper aims to highlight occupational-participation as an expansion of the international classification of functioning (ICF)'s concept of participation, to encapsulate a holistic approach for better functional outcomes and occupational wellbeing in the emerging field of cancer survivorship.

Occupational-participation, a central construct in the field of Occupational Therapy, is the person's engagement in work, play and daily living which is necessary for health and well-being. Cancer survivors can be empowered toward healthy occupational-participation to attain a healthy, 'health-after-cancer' status via a model of 'occupationalparticipation for cancer survivorship' (MOP.CS). The model is novel for addressing this missing link where its focus on occupational-participation living, can be used for designing seamless intervention programs for the connection to a "healthy-although-ill" living for cancer survivors.
\end{abstract}

Keywords: Cancer survivorship; ICF; Occupational-participation; Model of occupational participation for cancer survivors; Occupational therapy

\section{Introduction}

In recent year, mortality rate from cancer have declined between $20-26 \%$ [1] with improved survival rates which vary between 40 and 80 percent in low and high income countries respectively. With earlier detection and better treatment, the cancer survival rates have improved steeply. Among adults diagnosed with cancer during the period from 1974 through 1976, the 5-year relative survival rate for all cancers combined was $50 \%$, and the rates for common cancer include, breast, $75 \%$; prostate, $69 \%$; lung, $13 \%$; colorectal, $51 \%$; and bladder, $74 \%(\mathrm{NIH}$, 2010) [2,3]. Chronic disease is defined as a disease which has one or more of the following characteristics: "i. permanent and leaves residual disability; ii) caused by non-reversible pathological alternation; iii) requires special training of the patient for rehabilitation, or may be expected to require a long period of supervision, observation, or care" [4]. Based on these features, some cancer are increasingly being acknowledged as taking a form of chronic illness (a term used interchangeably with long-term conditions), requiring a care approach that extends beyond mere medical care into wider daily aspects of psychosocial-emotional and occupational functioning or participation for an indefinite period of time. With a longer course duration, the evidence-based management of these long-term conditions calls for high priority focus on active patient participation to be embedded in the delivery of health care models, and to the importance of selfmanagement $[5,6]$.

Previously, people living with cancer were considered cancer survivors if they had remained free of disease for five or more years after diagnosis $[7,8]$. However, advances in early detection and treatment mean that more cancers are curable, whilst, some have become chronic illnesses-i.e. not curable but can be controlled and managed over indefinite period of time. Cancer survivorship is now defined as the period from the time of diagnosis until the end of life (National Cancer Institute). The National Coalition for Cancer Survivorship echoed a similar definition-i.e. "from the moment of diagnosis and for the balance of life, an individual diagnosed with cancer is a survivor" (NCCS), but it covers family and caregiver. Regardless of its label cancer survivors need to be managed and self-manage beyond adjuvant-therapy periods, and across a myriad of survivorship issues. However, the traditional medical model of healthcare is not comprehensive because i) it tends not to acknowledge that the mind-body consequences post-cancer are critical on daily functioning, ii) addressing only the obvious physical impairments, and iii) prolonging a care focusing predominantly on cure, mere survival-emphasis, $[9,10]$. Non-medical tasks such as ability to resume life occupation, engagement in meaningful activities after cancer etc. are equal contributor and possibly independent factor for quality of life issues, are still neglected in occupational rehabilitation. In addition, patients' self-management skills are also often neglected, within the medical model of care [11]. These gaps need to be addressed now as many cancers adopt a chronic condition status.

There are emerging models of survivorship care to address the gap in care. A recent systematic review identified nine empirical studies of survivorship care models, found substantial variation and heterogeneity (optimal nature, timing, intensity, format, and outcomes) across these care models, and calls for further research [12]. This include model to address the gap not highlighted in these papers. Indeed, the potential fragmental care, also due to anticipated shortages in the current oncology workforce which requires more nurse

*Corresponding author: Loh SY, Department of Rehabilitation, Faculty of Medicine, University of Malaya, 50603 Kuala Lumpur, Malaysia, Tel: +794946678; E-mail: syloh@ummc.edu.my

Received February 24, 2016; Accepted July 27, 2016; Published July 29, 2016

Citation: Loh SY, Jonsson H (2016) Cancer Survivorship Care: A perspective from an Occupational-Participation Approach. J Cancer Sci Ther 8: 179-184. doi:10.4172/1948-5956.1000411

Copyright: (c) 2016 Loh SY, et al. This is an open-access article distributed under the terms of the Creative Commons Attribution License, which permits unrestricted use, distribution, and reproduction in any medium, provided the original author and source are credited. 
practitioners, but also more occupational therapists in order to address the occupational-participation gap in this shared care. Collaborative partnership with primary care providers can better deliver seamless survivorship care to the rising rates of survivors, who are living longer, and would need intervention related to occupational functioning and participation.

There are certainly gaps in care, that have not been explored nor studied, but the fact remains that more are needed to support people in the uncharted field of cancer survivorship phase. Even in more practical issue like return-to-work for the rising number of cancer survivor, there were gaps in the knowledge base of models to address these return-to-work issues [13]. More research work in this theoretical area is needed, as understanding the role of 'occupation' (everyday living activities) in the current self-management and prevention direction of health services within cancer as chronic illnesses, is grossly lacking. An occupational-participation perspective to cancer survivorship may help address the missing link for a holistic care.

\section{Aim and structure of paper}

In this paper, occupational-participation is used as an expanded and specified concept of participation in the international classification of functioning (ICF), to encapsulate a holistic approach for cancer survivorship care for the individual, as they attempt to readjust from a period of living with illness, into another indefinite stage of livinga-healthy-although-ill life. The aim of this paper is to discuss the consequences of cancer condition for health care, with an occupationalparticipation perspective that can better acknowledge cancer as a form of chronic illness in need for a rehabilitation approach targeted to promote participation in everyday life.

\section{The burden of cancer survivorship}

A cancer survivor is a person who has been diagnosed with cancer, from the time of diagnosis through to the end of initial treatment, the transition from treatment to extended survival, and long-term survival [13]. Cancer survivors are at risk for multiple severe and persistent symptoms, but these late and long-term effects are understudied [14]. With longer survivorship, greater efforts to address the many neglected and new physical-psycho-social-occupational functioning consequences are now needed. These functional domains of living are all important sequela with influential impact on the quality of life of cancer survivors. They also represent the subjective experience of participation in life situations which have been eminently missing from the traditional model of cancer care.

Overall, the vast majority of cancer survivors have one or more side effects during treatment [15], where pain and fatigue [16] followed by impairment in emotional functioning, anxiety, depression, insomnia and body image [17]. Cancer-related fatigue interferes with usual activities. Almost all patients receiving chemotherapy or radiation therapy experience fatigue [18], an underdiagnosed, underreported, and undertreated side effect. Another under-reported symptom is emotional distress, affecting almost half of all cancer patients $[19,20]$. Treatment related cognitive impairments is also a new area gaining recognition as more women report its negative impact on daily functioning and participation. A diagnosis of cancer can also hinder women from working, for a minimum of a few months to years, whilst some women may never return to work. A recent survey showed that almost 70 percent of previously employed breast cancer survivors did not return to work after 12 months post diagnosis. The reasons were wide ranging, from fears, myths, physical-psychological disabilities, as well as more positive reasons like revaluing life and life priorities [21].

Cancer like many other chronic illnesses has debilitating impact on many areas in a patient's daily life. Firstly, impairment and disability arising from cancer is wide ranging and has impact on a person's functional status and quality of life. Secondly, the disability in cancer like breast and internal organs, to some extent is rather invisible, unlike the visually crippling disability in more physical-form chronic conditions like arthritis and stroke. Thirdly, in terms of duration, although impairment may not be permanent, the impact from living with uncertainty for a long duration is a real burden, and the subjective interpretations can often be taxing on the person's quality of life. Health literacy research on patients' ability to obtain and understand information and services relevant to appropriate health decision-making $[22,23]$ is also poorly coordinated. Low health literacy contributes to poor treatment compliance, uncontrolled disease, and high healthcare utilization. Contrary adequate, relevant information facilitate decisionmaking, coping and psychosocial adjustment $[24,25]$. Women's health can be negatively affected by lack of education, inadequate information and a lack of awareness of the factors contributing to the disease [26], leading to feelings of helplessness and a sense of loss and distress [27]. Even, treatment decision difficulties, defined as difficulty in making decision about treatment, have been shown to predict persistence of psychological morbidity following breast cancer surgery [25]. A model of care that is address these issues either as a personal or environmental barrier which impedes or enhance participation in everyday life, is needed.

\section{Concept of participation in ICF}

The International Classification of Functioning, Disability and Health or ICF (WHO) is a theoretical framework to inform interprofessional collaboration of the health concept. As a biopsychosocial model, the ICF framework with uniform terminology enables common descriptions of the impact of a disease at the body-structure, activity and participation level, and facilitates collaboration of related health disciplines (Figure 1).

This approach takes a positivist stand (from the earlier ICF with a predecessor view taken from disability studies), where the ICF's

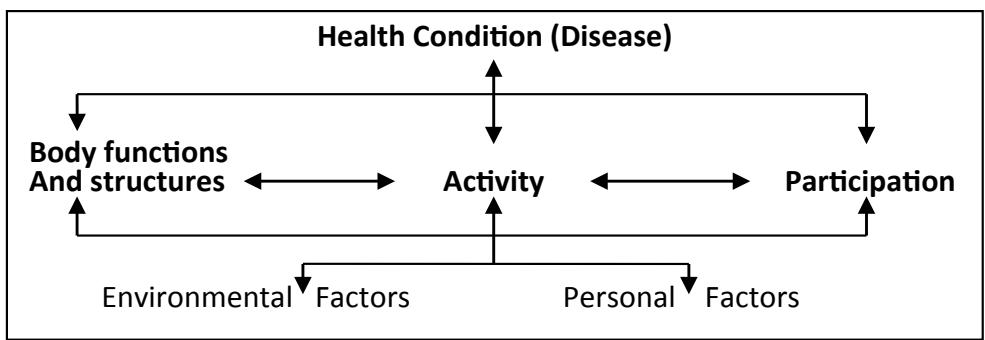

Figure 1: ICF Bio-psycho-social model of health (WHO). 
concept of participation differ from the original ICHD's focus on a lack-of-ability - in tandem with a traditional medical model. This newer approach focuses on ability and as such, asserts the rights of disabled people to participate fully in the society $[25,26]$. The ICF has a proactive shift-from a 'disease' classification to a positive 'health' classification. Previously, it emphasized the malfunctioning biological body as the primary determinant of disability, but, the new ICF interpret disability with a broader interdisciplinary view of the biological body, the social and the institutional structures [26,27]. As an illustration, ICF's define 'participation', as “a person's involvement in life situations", a concept of participation that may still be rather nebulous. Thus, in order to help answer what exactly is, 'involvement in life situation', we will address this gap with literature from occupational therapy.

\section{Occupational-participation-the link to well-being and life after cancer}

The concept of occupation is defined as the activities of daily living by the individuals in their environment [28]. Meaningful activity is thus, the broader existential aspect of the occupation for the person [29]. Thus, for cancer survivors, their renewed motivation, readjusted interests, and lifestyle habits may exert an influential drive towards selfmanaging a lifestyle to reduce risk. This concept underlined theories of occupational therapy that a balance of different occupations is beneficial to health and well-being, and balance is defined in terms of the time spent in self-care, productivity and leisure [30].

Occupational-participation has be defined as: "engaging in work, play or activities of daily living that are a part of one's sociocultural context and that are desired or necessary to one's well-being". Thus, occupational-participation connotes, "doing things with personal and social significance" [28]. So, how is this important in cancer survivorship model of care? There is a dire need for more research to discuss the importance of occupational-participation for people living with cancer, but a framework to guide the proposed comprehensive care is clearly needed and it's timely.

Successful occupational-participation depends on the barriers and facilitators within the environmental context. In particular, variations are attributed to the environmental differences in diet and lifestyle, are important for cancer control. Timely efforts are needed to identify and implement interventions that are aim at enabling health of cancer survivors. At present, research evidence is highlighting that chronic diseases may best be managed by a balance of traditional medical care and the day-to-day practice of self-management skills [31-34]. Therefore, it is timely to understand cancer survivorship from the perspective of 'occupational-participation', as factors acting as barrier or facilitator to execute the performance can be delineated and studied. The ICF model allows the therapists' views in terms of managing change in functional status in activities of everyday living; and allows the perspective of the survivors as the 'expert-patients'. This concept of occupation is inherently subjective and addresses the individual view of their meaningful 'daily activities' that they need to sustain for their wellbeing. Therefore, applying an occupational-participation perspective to the framework will guide therapists to focus on the daily things that people do, want to do, as well as, the significant meaningful occupation for building a new lifestyle-taking into consideration a new living norm with conscious input of cancer prevention, healthpromotion and wellness after cancer [35-37].

\section{Creating connections back to life}

Among cancer survivors who are "healthy although ill", occupational-participation were interpreted from their narrations as the therapeutic media 'experienced as links to life'. After a period of activity disruption due to acute cancer treatment, going back to usual daily activities offer the needed "routines and continuity" which was identified as a safe, familiar framework stimulating participants' everyday agency [38]. Thus, these uses of occupational activities creates the essential connections back to life, which have been evident in many conditions, but are somewhat less clear for cancer survivors.

\section{Creating connections for wellbeing and quality of life during the indefinite period}

People diagnosed with cancer spend quite a period of time undergoing treatment where there are often significant disruptions in their everyday life. Disrupted activities engagement caused by the loss of bodily part and/or functions, the loss of social roles, lowers a survivor's capacity to engage in basic daily activities [39]. With increasing evidence of longer survivorship [40,41], the concept of 'creating connection to life' through daily occupation, should be pursued one step further. It is timely to increase the emphasis on improving the well-being and quality of life of cancer survivors through the concept of patient's self-management, health-promotion (e.g. via attaining a healthy body weight and be physically active) and risk reduction [42-44]. Occupational engagement with informed meaningful and healthy choices, would thus, contributes to longer and better quality of life and it is attainable for cancer survivors today. In addition, the concept of empowerment as a psychological state that can act as a determinant of consequent participation and selfmanagement, is critical. Empowerment has been described with the concepts self-efficacy, active patient participation, increasing abilities, and control of life. Empirical evidence suggests a positive relationship between empowerment and long-term health outcomes [45]. With the concept of empowering survivors embedded in this model of care, the functional goal of rebuilding healthy occupations for a longer (albeit indefinite) and a healthier (despite being ill), independent living to its fullest may be more attainable.

Cancer survivors must be alerted to make conscious changes in their new lifestyle habits, in order to promote risk reduction habits. Therefore, getting patients to re-focus on building health (rather than looking for complete 'cure') is the direction since rates of survival has increased dramatically with better treatment [46]. Addressing occupational-participation provides the researchers and the therapists, a more integrated, meaningful approach for cancer survivorship care, in order to facilitate survivors to create meaning during the period of indefinite living-successfully. During this uncertain period, occupational engagement allows survivors to create connections to daily life and enlarge the experience of self as an active (living) person. Indeed, occupational perspective where used of occupations as intervention represent "normal" life (targeting a healthy new norm) for cancer survivors within and outside the healthcare system.

Occupational Therapy for cancer conditions focuses on a primary prevention goal of enhancing healthy lifestyle; and risk-reduction habits to keep cancer at bay, along daily occupational-participation to improve length and quality of life. Our rehabilitation aim must now extend beyond reducing present disabilities (visible and invisible ones) towards more concerted efforts on facilitating improvement in participation and quality of life. Therefore, a comprehensive management covering health, emotions and roles as well as managing risk-reduction, play a crucial part in the overall management of cancer survivors [46], as in many chronic illnesses. The role of preservation, 


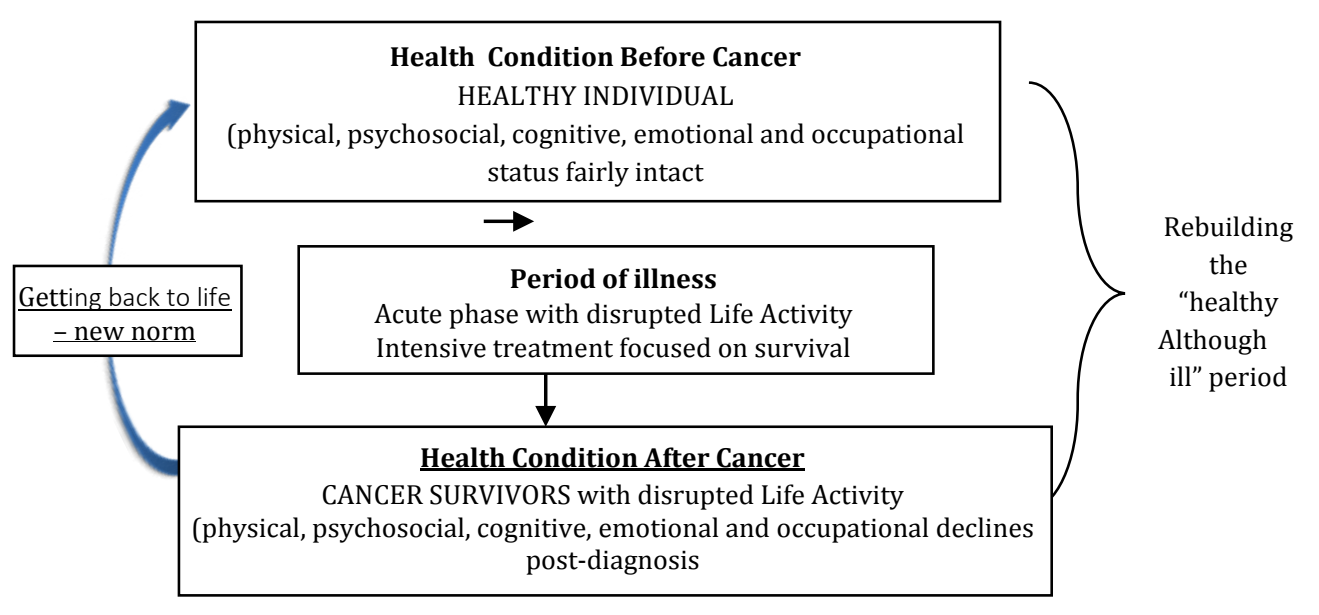

Empowerment via Occupational Participation

(re)-engaging in work, daily living activities, leisure

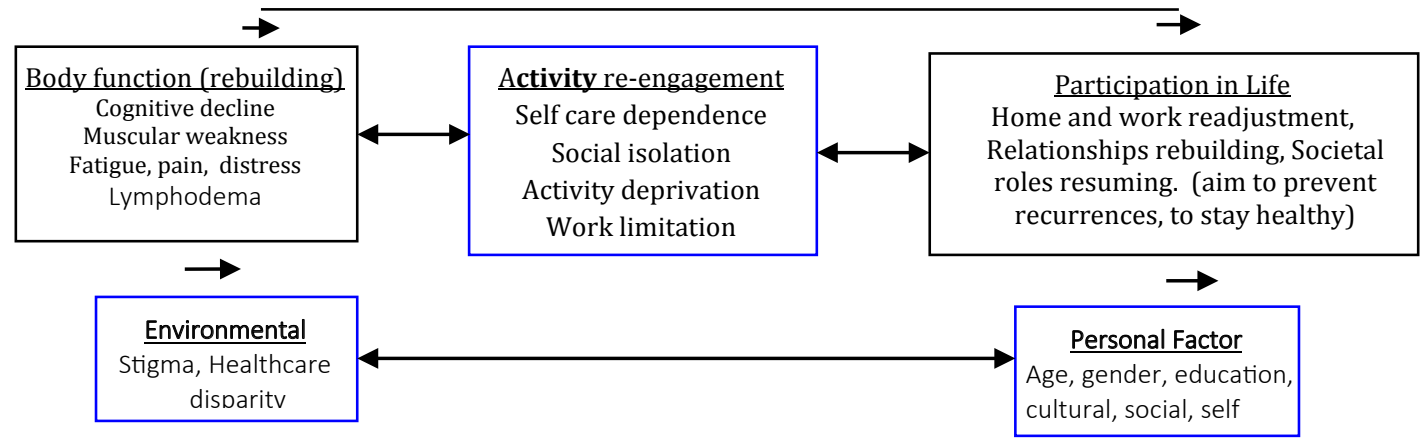

Figure 2: Model of Occupational-participation for cancer survivorship (MOPCS). Authors expansion of the ICF-model (lower part of the figure) by promoting empowerment through occupational-participation' to create connections back to "life".

reframing, readjustment and re-enactment of successful occupationalparticipation, depends on both personal and environmental factors, to achieve mastery and enabling-experiences which build up individual's self-determination, self-efficacy and autonomy. This is because throughout the experience of cancer and its associated treatment changes to a person's values, the perspectives on occupations shifted as they engages in meaningful reflections to find meanings to life situations in order to adapt to their situation $[47,48]$. The model inform a direction of care where an occupational perspective acknowledges the environment as complex phenomena that play several congruous but also sometimes incongruous roles.

In cancer survivorship care, encapsulating a continuum of behavioural care (curative, rehabilitative as well as preventive healthcare) for survivors is timely. It should begin early throughout the four essential component of survivorship care, in order to maximize cure, optimize the recovery process and proactively prevents all aspects of disabilities. The IOM (Institute of Medicine) [49-51] report noted these four essential components of survivorship care as: i) prevention and detection of new cancers and recurrent cancer; ii) surveillance for cancer spread, recurrence, or second cancers, iii) intervention for consequences of cancer and its treatment, and iv) coordination among specialists and primary care providers (PCPs) to ensure that all of the survivors health needs are met (e.g., health promotion, immunizations, screening for cancer and noncancerous conditions, care of concurrent conditions). Nevertheless it's also proactive, to consider perspectives from a cellular dimension. Research has shown that imprinted DNA methylation [established during gametogenesis in the parental germ line [52], and their associated loss of imprinting [LOI]) have been observed in a large variety of human carcinomas [53]. Thus, survivors should be followed up for any imprinted gene associated diseases. Future study could check if these survivors express normal levels of enzymes required for the maintenance of imprinting DNA methylation- e.g. DNA methyl-transferases (DNMTs) and histone methyl-transferases (HMTs) [54,55]. Survivors who have occupationally-active participation can be followed on the level of enzyme to examine for better wellness and lower cancer recurrences.

Figure 2 is an attempt to present the concept of occupationalparticipation in living indefinitely with cancer, as an expansion of the ICF model applied to cancer survivorship care. The IOM's four component of survivorship care can be represented in the box 'health condition after cancer' and a vertical theme that runs across these components is an occupational-participation theme. We proposed the hypothetical associations that encapsulate the core ICF for a better engagement of occupational-participation, and a call for a more comprehensive focus on participation at the 'health-after-cancer' state. The preservation, adaptation and re-adjustment of new norm and a healthy participation to rebuild health and prevent recurrences is now needed to address this gap that is widening with more and more 
survivors living longer, but having to face the numerous side-effects, new emerging issues surrounding living longer with cancer.

\section{Conclusion}

The ICF general framework used to conceptualize comprehensive care for cancer survivors in a general sense is not holistic enough, as survivors' occupational-participation is clearly missing, and needs to be embedded and defined. This gap that we identified can be addressed from an occupational-participation perspective. As a form of longterm illness, many cancers are comprehensive in their effects, as well as being tremendously varied from one person to another. The health implication is far more than merely treating the illness. After the acute phase with often heavy 'survival-focused' treatment, clients are in need for professional guidance in their way back to a "healthy-although-ill" life. Preservations techniques need to be learned, whilst, adaptation might be necessary in their homes, work-places or community support projects. In addition, readjustments towards life's important tasks can be enabled via supportive care programs held in the community. An example of this approach is the Petaling Jaya's Keep Able cancer community founded to support and enable cancer survivors to redesign lifestyle [49]. Another example is an ongoing RCT-study in Denmark applying an occupational therapy approach in a tailored intervention in the home environment for persons with cancer [51]. Occupational Therapists are the most suitable health professionals for these daily activities tasks. Along with the engagement of these therapists, a view of integrated services for the current care of cancer survivors is needed if health organizations are genuine about providing effective seamless community reintegration and social preventive public health interventions. Of course other professionals' specific inputs are also needed in an inter-professional approach, one which consider the subjective view of survivors, and incorporate patient selfmanagement, as the means to empower them to be more active in care. This also augurs well for a new approach in health care to better serve the extended needs of a rising population of cancer survivors. An occupational-participation perspective allows health professionals a better approach to manage cancer condition that can lasts for years or decades. This model is novel with its focus on occupationalparticipation for functional living and can be used for designing seamless intervention programs for connection to a "healthy-althoughill" living for cancer survivors. The effectiveness of cancer survivorship care warrants a dire change, not just in the roles of our patients but in the whole approach and attitudes of health professionals for a more integrated care, as well as the views and engagement of the public as a caring community.

\section{Funding}

This work is partly funded by University Malaya Sustainability Grant 2016 awarded to the first author

\section{References}

1. Malvezzi M, Bertuccio P, Levi F, La Vecchia C, Negri E (2014) European cancer mortality predictions for the year 2014. Ann Oncol 25: 1650-1656.

2. International Agency for Research on Cancer (2012) Estimated cancer incidence, mortality, prevalence and disability-adjusted life years (DALYs) worldwide in 2008. Globocon 2008, France.

3. NIH (2010) Fact Sheet Cancer. National Cancer Institute. Accessed on: August $01^{\text {st }}, 2016$.

4. DHA (1998) Delaware Healthcare Association Glossary of Health Care Terms and Acronyms. Dover, Delaware: Delaware Healthcare Association.

5. Donaldson $L$ (2003) Expert patients usher in a new era of opportunity for the NHS. BMJ 326: 1279-1280.
6. Tyreman S (2005) The Expert patient: outline of UK government paper. Med Health Care Philos 8: 149-151.

7. Twombly R (2004) What's in a name: Who is a cancer survivor?. J Natl Cancer Inst 96: 1414-1415.

8. Leigh S (2007) Cancer Survivorship: A Nursing Perspective. In: Ganz P (Ed) Cancer Survivorship: Today and Tomorrow. Springer, New York, NY.

9. Bell K, Ristovski-Slijepcevic S (2013) Cancer Survivorship: Why Labels Matter $\mathrm{J}$ Clin Oncol 31: 409-411.

10. Loh SY, Yip CH (2006) Breast cancer as a chronic illness: Implications for Rehabilitation and Medical Education. Jummec 9: 3-11.

11. Loh SY, Packer T, Yip CH, Low WY (2007) Perceived barriers to selfmanagement in Malaysian women. Asia Pac J Public Health 19: 52-57.

12. Désiron HAM, Donceel P, de Rijk A, van Hoof E (2013) Return to work following breast cancer. J Occup Rehabil 23: 516-526.

13. Mullan $F(1985)$ Seasons of survival: reflections of a physician with cancer. N Engl J Med 313: 270-273.

14. Burkett VS, Cleeland CS (2007) Symptom burden in cancer survivorship. Cancer Surviv 1: 167-175.

15. Barbera L, Seow H, Howell D, Sutradar R, Earle C, et al. (2010) Symptom burden and performance status in a population-based cohort of ambulatory cancer patients. Cancer 116: 5767-5776.

16. Patrick DL, Ferketich SL, Frame PS, Harris JJ, Hendricks CB, et al. (2002) National Institutes of Health State-of-the-Science Conference Statement: Symptom management in cancer: pain, depression, and fatigue, July 15-17, 2002. J Natl Cancer Inst 32: 9-16.

17. Knobf MT, Sun Y (2005) A longitudinal study of symptoms and self-care activities in women treated with primary radiotherapy for breast cancer. Cancer Nurs 28: 210-218

18. National Comprehensive Cancer Network (2011) Clinical Practice Guidelines in Oncology (NCCN Guidelines) Cancer-Related Fatigue Version 1.2011.

19. Zabora J, BrintzenhofeSzoc K, Curbow B, Hooker C, Piantadosi S (2001) The prevalence of psychological distress by cancer site. Psychooncology 10: 19-28.

20. van den Beuken-van Everdingen MH, de Rijke JM, Kessels AG, Schouten $\mathrm{HC}$, van Kleef M, et al. (2007) Prevalence of pain in patients with cancer: a systematic review of the past 40 years. Ann Oncol 18: 1437-1449.

21. Tan FL, Loh SY, Su TT, Veloo VW, Ng LL (2012) Return to work in multi-ethnic breast cancer survivors - a qualitative inquiry. Asian Pacific Journal of Cancer Prevention 13: 5791-5797.

22. CDC (2012) Chronic Diseases and Health Promotion. National Center for Chronic Disease Prevention and Health Promotion. Accessed on: August 01 ${ }^{\text {st }}$ 2016.

23. Healthy People 2010 (2006) Health Communication II. In: Nielsen-Bohlman (Ed) 2004 Health Literacy: A Prescription to End Confusion. The National Academies Press, Washington DC.

24. Iconomou G, Viha A, Koutras A, Vagenakis A, Kalofonos H (2002) Information needs and awareness of diagnosis in patients with cancer receiving chemotherapy: a report from Greece. Palliative Med 16: 315-321.

25. Lam W, Chan M, Hung W, Fielding R (2007) Treatment decision difficulties and post-operative distress predicts persistence of psychological morbidity in Chinese women following breast cancer surgery. Psycho-Oncology 16: 904-912.

26. Luddy G (2007) Women, disadvantage and health. Irish Med J, 100: 71-73

27. Ankem K (2006) Use of information sources by cancer patients: results of a systematic review of the research literature. Information Research 11.

28. Kielhofner G (2008) Model of Human Occupation: Theory and Application. Lippincott Williams \& Wilkins, Baltimore, MD

29. Nelson D (1988) Occupation: Form and performance. Am J Occup Ther 42 633-641.

30. Doble SE, Santha JC (2008) Occupational well-being: Rethinking occupationa therapy outcomes. Can J Occup Ther 75: 184-190.

31. World Health Organization (2001) International classification of functioning disability and health (ICF). Geneva, Switzerland 
Citation: Loh SY, Jonsson H (2016) Cancer Survivorship Care: A perspective from an Occupational-Participation Approach. J Cancer Sci Ther 8: 179-184. doi:10.4172/1948-5956.1000411

32. Moschetti I, Cinquini M, Lambertini M, Levaggi A, Liberati A (2000) Follow-up strategies for women treated for early breast cancer. Cochrane Database Syst Rev 27: CD001768.

33. Parkin DM, Bray F, Ferlay J, Pisani P (2005) Global Cancer Statistics, 2002. CA Cancer J Clin 55: 74-108.

34. Von Korff M, Gruman J, Schaefer J, Curry SJ, Wagner EH, et al. (1997) Collaborative management of chronic illness. Ann Intern Med 127: 1097-1102.

35. Barnes C, Mercer G, Shakespeare T (2000) Exploring disability: A sociological introduction. Cambridge, England: Polity

36. Hurst $R$ (2003) The international disability rights movement and the ICF. Disabil Rehabil 25: 572-576.

37. Imrie R (2004) Demystifying disability: A review of the international classification of functioning, disability and health. Sociology of Health \& Illness 26: 287-305.

38. la Cour K, Nordell K, Josephsson S (2009) Everyday Lives of People With Advanced Cancer: Activity, Time, Location, and Experience. OTJR: Occupation, Participation and Health 29: 154-162.

39. Taylor K, Currow D (2003) A prospective study of patients' identified unmet activity of daily living needs among cancer patients at a comprehensive cancer care centre. Australian Occupational Therapy Journal 50: 79-85.

40. De Santis CE, Lin CC, Mariotto AB, Siegel RL, Stein KD, et al. (2014) Cancer treatment and survivorship statistics CA: A Cancer Journal for Clinicians 64 252-271.

41. IARC (2013) International Agency for Cancer Research. Latest world cancer statistics. Accessed on: August $02^{\text {nd }} 2016$.

42. Institute of Medicine (2006) From Cancer Patient to Cancer Survivor: Lost in Transition. The National Academic Press.

43. La cour K, Johannessen $\mathrm{H}$, Josephsson S (2009) Activity and meaning making in the everyday lives of people with advanced cancer. Palliative and Supportive Care 7: 469-479.

44. Danaei G, Vander Hoorn S, Lopez AD, Murray CJ, Ezzati M (2005) Causes of cancer in the world: comparative risk assessment of nine behavioural and environmental risk factors. Lancet 366: 1784-1793

45. Rogers ES, Chamberlin J, Ellison ML, Crean T (1997) A consumer-constructed scale to measure empowerment among users of mental health services. Psychiat Serv 48: 1042-1047.

46. Loh SY, Yip CH (2006) Breast cancer as a chronic illness: Implications for Rehabilitation and Medical Education. Jummec 9: 3-11.

47. Hoppes S (2005) When a child dies the world should stop spinning: an autoethnography exploring the impact of family loss on occupation. Am J Occup Ther 59: 78-87.

48. Forhan M (2010) Doing, being, and becoming: a family's journey through perinatal loss. Am J Occup Ther 64: 142-151.

49. SY Loh (2016) Community support for cancer patients. Accessed on: August 2nd 2016.

50. Halpern MT, Viswanathan M, Evans TS, Birken SA, Basch E, et al. (2015) Models of Cancer Survivorship Care: Overview and Summary of Current Evidence. J Oncol Pract 346.

51. Brandt A, Pilegaard MS, Oestergaard LG, Lindahl-Jacobsen L, Sørensen J et al. (2016) Effectiveness of the "Cancer Home-Lifelntervention" on everyday activities and quality of life in people with advanced cancer living at home: a randomised controlled trial and an economic evaluation. BMC Palliative care 15: 1-11.

52. Wood AJ, Oakey RJ (2006) Genomic Imprinting in Mammals: Emerging Themes and Established Theories. PLOS Genetics 2: e147.

53. Jelinic $P$, Shaw $P$ (2007) Loss of imprinting and cancer. J Pathol 211: 261-268.

54. Vilkaitis G, Suetake I, Klimasauskas S, Tajima S (2004) Processive methylation of hemimethylated $\mathrm{CpG}$ sites by mouse Dnmt1 DNA methyltransferase. J Bio Chem 280: 64-72.

55. Zhang T, Termanis A, Özkan B, Bao XX, Culley J, et al. (2016) G9a/GLP Complex Maintains Imprinted DNA Methylation in Embryonic Stem Cells. Cell Rep 15: 77-85. 